\title{
Color Coded Perfusion Imaging with Contrast Enhanced Ultrasound (CEUS) for Post-Interventional Success Control Following Irreversible Electroporation (IRE) of Primary and Secondary Malignant Liver Lesions
}

\author{
Janine Rennert $^{1^{*}}$, Isabel Wiesinger ${ }^{1^{*}}$, Andreas Schicho ${ }^{1}$, Philipp Wiggermann ${ }^{1}$, Christian Stroszczynski ${ }^{1}$, Lukas Philipp \\ Beyer $^{2}$, Ernst Michael Jung ${ }^{3}$
}

1) University Hospital

Regensburg, Department of

Radiology, Regensburg

2) University Hospital

Regensburg, Center for

Radiological Interventional

Oncology, Regensburg

3) University Hospital

Regensburg, Department

of Ultrasound, Regensburg,

Germany

Address for correspondence:

Janine Rennert, MD

Department of Radiology

University Hospital

Regensburg

Franz-Josef-Strauss Allee 11

93053 Regensburg

Germany

jarennert@yahoo.de
Received: 04.07.2019

Accepted: 23.08.2019

* contributed equally to this work

\section{ABSTRACT}

Aim: Evaluation of the post-interventional success following irreversible electroporation (IRE) using a new color coded perfusion quantification software with contrast-enhanced ultrasound (CEUS) in patients with malignant lesions of the liver.

Methods: Thirty-eight patients with 68 malignant liver lesions underwent IRE. All malignant lesions were investigated with CEUS before and within 24 hours following IRE to detect residual tumor tissue. The parameters analyzed by color coded perfusion quantification software were: the peak enhancement $(\mathrm{pE})$, time to peak (TTP), mean transit time (mTT), rise (Ri) and wash-in area under the curve (WiAUC). Perfusion in the center, the margins of the lesions and in the surrounding liver were evaluated using these parameters. Results: Hepatocellular carcinoma (HCC) with complete ablation showed significantly different changes between the center and the margin of the lesions for WiAUC $(\mathrm{p}<0.05)$ and $\mathrm{pE}(\mathrm{p}<0.01)$. Also significant differences were noted between the center of the lesions and the surrounded tissue for the same parameters $(\mathrm{p}<0.01)$. In the completely ablated metastatic lesions, significant differences were found between the center of the lesion and the margins $(\mathrm{p}<0.01)$ and between the center of the lesion and the surrounding liver $(\mathrm{p}<$ 0.05 ) for WiAUC. mTT, TTP and Ri showed no significant changes between the center of the lesions, margin of the lesions or surrounding tissue. Also, no significant differences were found for these parameters in the different regions of interest for HCC or the metastatic lesions with partial ablation success.

Conclusion: CEUS with perfusion imaging is a valuable supporting tool for the post-interventional evaluation of liver lesions following IRE. Focus should be placed on the peak enhancement (pE) and the wash-in area under the curve (WiAUC).

Key words: irreversible electroporation - contrast enhanced ultrasound - perfusion analysis - hepatocellular carcinoma.

Abbreviations: CCDS: Color Coded Doppler Sonography; CEUS: contrast-enhanced ultrasound; CT: computer tomography; HCC: hepatocellular carcinoma; IRE: irreversible electroporation; MRI: magnetic resonance imaging; mTT: mean transit time; MWA: microwave ablation; PD: Power Doppler; pE: peak enhancement; RFA: radiofrequency ablation; Ri: rise; TTP: time to peak; SIRT: selective internal radiation therapy; WiAUC: wash-in area under the curve.

\section{INTRODUCTION}

Liver malignancies are the 6th most common cancers worldwide, with approximately 750,000 new cases per year. They represent the 3 rd most common cause of cancer-related deaths worldwide. Hepatocellular carcinoma (HCC) accounts for over $90 \%$ of all primary liver cancers, having an increasing incidence and thus becoming a major healthcare problem [1]. Depending on the local tumor extent, extrahepatic dissemination and the established degree of liver damage, various therapeutic options are available for each stage. Thus, for early stage HCC, surgical resection [2], ablation [3] and transplantation [4] are recommended. For intermediate-stage HCC, trans-arterial chemoembolization (TACE) [5] is favored, for advanced-stage HCC sorafenib [6] and for terminal-stage HCC, the best supportive care [6].

The most common liver metastases are those of the gastrointestinal tract (especially the colon and the pancreas), breast and lung tumors. The tumor cells reach the liver 
through various paths, the portal system, the hepatic artery, the lymphatic vessels, or by direct extension from nearby organs [7]. The management of metastatic liver lesions has become more aggressive and is managed by multidisciplinary teams. In addition to the standard management represented by surgical resection and chemotherapy $[8,9]$, there are various new approaches: liver transplantation [10], selective internal radiation therapy (SIRT) using Yttrium 90, a particular $\beta$-transmitter with limited tissue penetration [11], radiotherapy and ablative techniques, such as radiofrequency ablation (RFA), irreversible electroporation (IRE) and microwave ablation (MWA).

Primary and secondary liver tumors bordering major hepatic blood vessels or biliary ducts are a relative contraindication to surgery, if a complete resection with sufficient liver remnant is not possible. Thermal ablative techniques such as RFA and MWA also have a high risk of thermal damages to adjacent or internal vital structures. Furthermore, the risk of local recurrence for lesions located near large blood vessels is higher, due to the so-called heat-sink effect [12].

Irreversible electroporation is a minimally invasive imageguided technique in which the application of ultrashort highvoltage electrical pulses leads to an irreversible increase in the permeability of the cell membrane, causing cell death [13]. It is indicated for both primary and secondary liver malignancies when surgical resection or other ablative techniques are not suitable. Irreversible electroporation offers several potential advantages over thermal ablation [14], particularly, the ability to treat tumors adjacent to large blood or biliary vessels and neural structures [15] by causing defects in the cell membranes of the tumor leading to irreversible permeabilization and finally cell death. However, when applied correctly, the surrounding tissue remains intact. One possible disadvantage of IRE is a lower success rate for complete local tumor eradication. Studies have reported local tumor progression rates of up to $40 \%$ at 12 months follow-up [16]. This might be explained by persisting difficulties regarding the optimal timing and imaging method during the procedure and for follow-up $[17,18]$.

Power Doppler (PD) or Color Coded Doppler Sonography (CCDS) allow the display of macrovascularization. Contrast enhanced ultrasound (CEUS) evaluates the microvascularization of the lesions and the new color coded perfusion quantification software allows quantitative measurement of microvascularization in a specific region of interest (ROI).

The aim of this study was to evaluate the post-interventional success following IRE using color coded perfusion quantification software with CEUS in patients with primary and secondary liver malignancies.

\section{METHODS}

\section{Study design}

From January 2013 until December 2017, 38 patients ( 9 females, 29 males, age range 46 - 82 years, mean 59.5 years) with 68 malignant liver lesions identified by characteristic imaging features and/or histopathology were included in this retrospective study. Each patient underwent pre-interventional CEUS and contrast-enhanced computed tomography (CT) or liver specific contrast enhanced magnetic resonance imaging (MRI) for detection and characterization of the liver lesions.

Exclusion criteria were contraindications for use of a contrast agent for CT, MRI or CEUS, impaired renal function (creatinine $>1.5 \mathrm{mg} / \mathrm{dl}$, creatinine clearance $<30 \mathrm{ml} / \mathrm{min}$ ), pre-existing strong allergic reactions and decompensated cardiac failure.

The therapeutic interventional procedure for each patient was a percutaneous IRE using a Nanoknife-Generator (Angiodynamics ${ }^{\oplus}$, Latham, NY, USA) for the ablation. This generator produces a voltage of up to 3000 volts and a current of up to 50 amperes. This energy is transmitted via the ablation electrodes into the target tissue in the form of very short electrical impulses. The ablation electrodes have an active tip of 5-20 $\mathrm{mm}$. The voltage necessary for the ablation is determined with the aid of a standard algorithm (Angiodynamics), which takes into consideration, among other things, the intended size of the ablation zone, the number of applied electrodes and the distance between them. All the interventions were performed under combined CT-fluoroscopy. In all patients, the treatment was performed under general anesthesia. The indication for local ablative therapy was decided at an interdisciplinary tumor board review.

Each patient was examined using fundamental B-mode, CCDS, PD and Contrast Enhanced Ultrasound (CEUS). The local Ethical Committee approved the use of CEUS for this study. Before the imaging procedures were conducted, written informed consent was obtained from each patient for MRI, CT and CEUS.

\section{Imaging studies}

\section{Basic ultrasound examination}

Within 24 hours following IRE, ultrasound was performed by an experienced radiologist with national DEGUM stage III using a multifrequency convex transducer (1-6 MHz, LOGIQ E9, GE Healthcare). First, the whole liver was examined using B-mode sonography in sweep technique. Color Coded Doppler Sonography and PD ultrasound were used to evaluate native vascularization. Flow parameters were adjusted to the lowest possible pulse repetition frequency $(\mathrm{PRF}<1000 \mathrm{~Hz})$ and the best possible color imaging without blooming artifacts.

\section{CEUS}

Contrast enhanced ultrasound was performed after bolus injections of $1-2.4 \mathrm{ml}$ of sulphur hexaflouride microbubbles (SonoVue ${ }^{\varpi}$, BRACCO, Italy) with a low mechanical index (MI $<0.16$ ) applying CEUS with amplitude modulation and pulse inversion harmonic imaging (PIHI) technique. The contrast harmonic imaging technique (CHI) uses a contrast-specific detection mode for real-time evaluation of the contrast-agent enhancement. The liver microcirculation was evaluated continuously from an early arterial phase (beginning $15 \mathrm{sec}$. after contrast application) until a late parenchymal phase (> 5 min.). During the first 60 seconds a short video clip was recorded. Afterwards until the late phase single images were digitally stored in PACS.

Irregular enhancement at the margins of the ablation defect during early arterial phase, preferably combined with a wash-out starting during portal venous phase was considered 
as a characteristic criterion for residual HCC tissue (Fig. 1). A uniform peripheral rim enhancement without wash-out was considered as a physiological postablation reaction (Fig. 2). Wedge-shaped, homogeneous arterial enhancement peripheral to the ablation defect with progressively enhancing portalvenous branching but without wash-out was defined as an arterio-portal-venous shunt (Fig. 3).

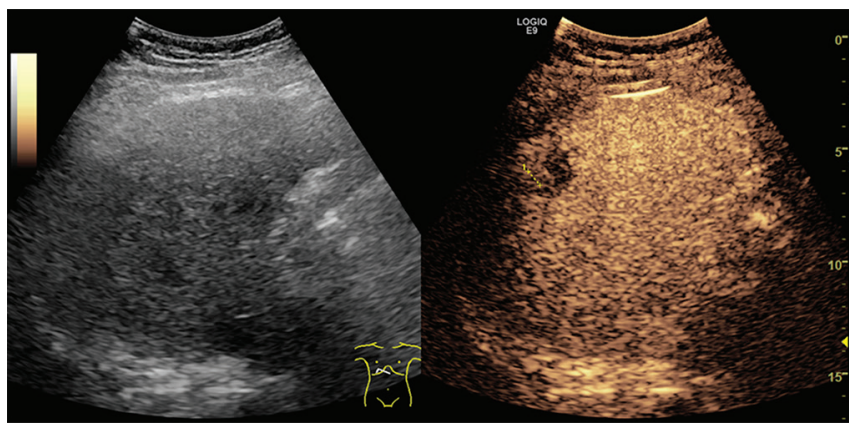

Fig. 1. CEUS image following IRE showing an irregular residual enhancement at the margins of the ablation defect, consistent with a residual tumor.
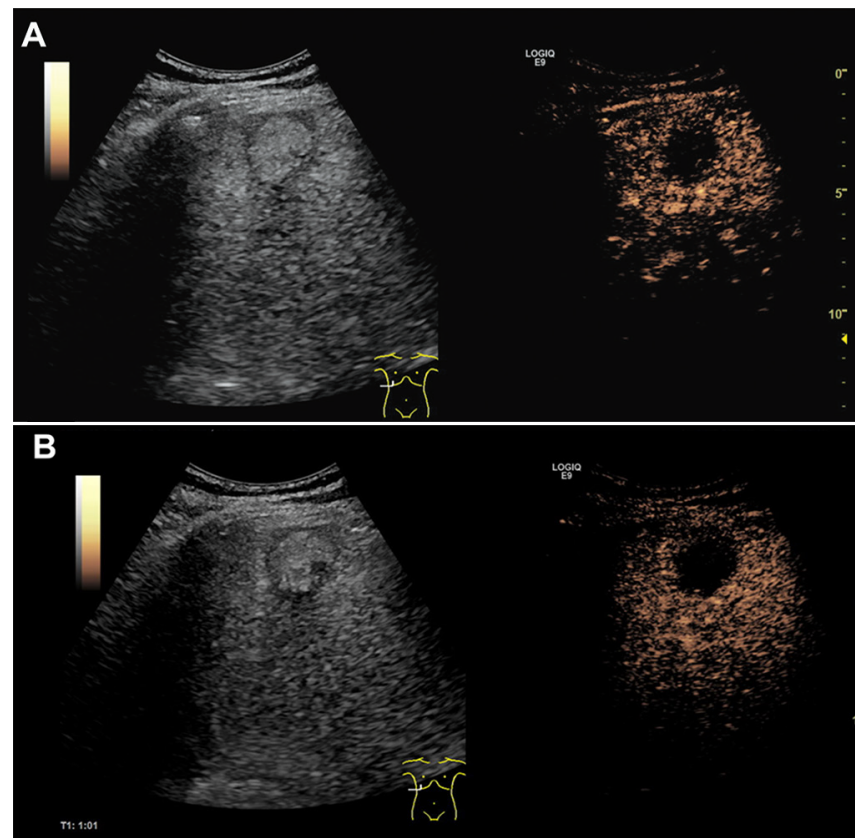

Fig. 2. CEUS image following IRE showing a uniform peripheral rim enhancement without wash-out (A: arterial phase after 24 sec., B portal venous phase after 1:01 $\mathrm{min}$ ), compatible with a physiological postablation reaction.

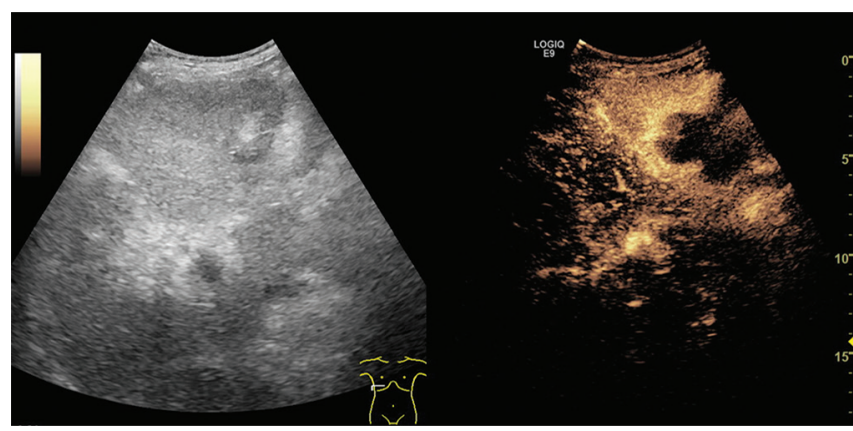

Fig. 3. CEUS image following IRE shows a wedge-shaped, homogeneous arterial enhancement peripheral to the ablation defect (24 sec.) with progressively enhancing portal-venous branching but without wash-out consistent with an arterio-portal-venous shunt.

\section{$C T / M R I$}

Before the intervention, every patient received a contrastenhanced CT (100-130 ml AccupaqueTM, GE) of the whole abdomen/ pelvis in portal-venous phase (70-90 sec). After the procedure only the liver was scanned in arterial (25-35 $\mathrm{sec}$ ) and portal-venous phase to exclude post-interventional complications. Each patient received an additional preinterventional contrast-enhanced MRI (Gd-EOB-DTPA, Primovist ${ }^{\oplus}$, Bayer, Schering Pharma AG, Germany) including $3 \mathrm{D}$ vibe sequences from arterial phase $(20-25 \mathrm{sec})$ up to late phase $(20 \mathrm{~min})$. Each patient underwent follow-up contrastenhanced MRI 1 day, 3 and 6 months following IRE.

\section{Perfusion analysis}

Static regions of interest (ROI) were manually placed in the center of the lesion, the margin and the surrounding tissue. The digitally stored ultrasound loops were evaluated using a color coded perfusion quantification software (VueBox ${ }^{\circledast}$, Bracco, Italy). Five parameters were calculated for each Region of Interest (ROI) which included time to peak (TTP), mean transit time (mTT), peak enhancement (pE), Rise (Ri) and Wash-in Area Under the Curve (WiAUC) [19,20]. Flow parameters such as regional blood volume and regional blood flow were calculated by the software and exported in a calculation protocol. In all but three lesions, each parameter was also estimated in the periphery.

\section{Image analysis}

Contrast-enhanced ultrasound examinations and the perfusion assessment were analyzed by two experienced radiologists in consensus. For each modality used, each observer recorded the diagnostic findings. Furthermore, the image quality was documented on a four points scale: 1 excellent, 2 - minor diagnostic limitations, 3 - major diagnostic limitations, 4 - non-diagnostic.

Imaging modalities were evaluated using the data analysis hard-/software of the ultrasound system (LOGIQ E9, GE). and external software (VueBox/BRACCO).

\section{Statistical analysis}

For calculation of the differences between the center of the lesion and the margin, the margin and the surrounding tissue as well as the center of the lesion and the surrounding tissue for each parameter repeated measures ANOVA with Bonferroni post test was performed using GraphPad Prism version 5.00 for Mac OS X, GraphPad Software, San Diego California USA with alpha $=0.05$ indicating statistically significant differences between groups.

\section{RESULTS}

\section{Lesions characteristics and tumor size}

Out of the 68 tumor lesions, 26 nodules (in 20 patients) showed an enhancement pattern consistent with HCC: arterial irregular hyperenhancement and portal venous to late washout on CEUS. Also, the pre-interventional imaging techniques showed characteristics compatible with HCC. The etiology of liver cirrhosis was alcohol consumption (17 patients) and viral hepatitis C (3 patients). The Child-Pugh Score was A in 13 patients, $B$ in 6 patients and $C$ in 1 patient. Model for end- 
stage liver disease (MELD) scores were within the range of 7 - 24. According to the Barcelona Clinic Liver Cancer (BCLC) algorithm for HCC staging and treatment 6 patients were in stage 0,9 patients in stage $A$ and 5 patients in stage $B$. In 16 out of 20 patients, a solitary HCC lesion was treated. In 2 patients, 2 and 3 HCC lesions were ablated, respectively.

The underlying malignancies for 42 lesions (in 18 patients) were metastases caused by colorectal carcinoma in 11 patients (28 lesions), by breast carcinoma in 2 patients (9 lesions), by neuroendocrine carcinoma in 2 patients ( 2 lesions), by prostatic carcinoma in 2 patients ( 2 lesions) and by cholangiocellular carcinoma (CCC) in 1 patient (1 lesion). In 6 patients, only one tumor lesion was treated using IRE. In 2 patients, the number of lesions treated was 2 , in 6 patients was 3 , in 2 patients was 4 and in 1 patient was 6 . If three or more lesions per patient needed to be treated using IRE, the intervention was carried out over the course of several days. The mean number of lesions treated in all patients was 2.5 .

The pre-interventional tumor sizes of the 68 lesions ranged from 11 to $55 \mathrm{~mm}$ (mean size $26 \mathrm{~mm}$ ). The postinterventional defects sized from 11 to $73 \mathrm{~mm}$ (mean size $38 \mathrm{~mm}$ ). In all lesions, a post-interventional reduction of the tumor microvascularization was observed. Out of the 68 lesions treated with IRE, in 53 lesions a complete ablation could be verified during follow-up of up to 6 months. A partial ablation success was confirmed in 15 lesions (7 HCC and 8 metastases). These patients were evaluated for a secondary procedure by the interdisciplinary tumor board. No major complications were noted.

\section{Perfusion analysis and CEUS}

In all 38 patients CEUS was feasible. The image quality in all examinations was excellent or had only minor diagnostic limitations (1-2 SD \pm 0.397$)$.

For evaluation of the main perfusion parameters $\mathrm{pE}$, WiAUC, mTT and TTP, 66 out of 68 lesions were included. For evaluation of Ri, only 44 lesions could be used as not all data was obtained. Regarding metastases with incomplete ablation success (8 lesions), no significant differences were found for each of the perfusion parameters (Fig 4, Table I).

Table I. Perfusion parameters for the ROIs in the center of the lesion, the margin and the healthy liver, acquired within $24 \mathrm{~h}$ following IRE metastases incomplete ablation success.

\begin{tabular}{lccc}
\hline & $\begin{array}{c}\text { Lesion } \\
(\text { mean } \pm \text { SD })\end{array}$ & $\begin{array}{c}\text { Margin } \\
(\text { mean } \pm \text { SD })\end{array}$ & $\begin{array}{c}\text { Surrounding } \\
\text { Liver } \\
(\text { mean } \pm \text { SD })\end{array}$ \\
\hline pE (dB) & $834.2 \pm 1564$ & $2708 \pm 3855$ & $2176 \pm 2527$ \\
WiAUC (-Inf.) & $1191 \pm 2767$ & $1701 \pm 1701$ & $976.6 \pm 1314$ \\
$\operatorname{mTT}(\%)$ & $109.7 \pm 199.9$ & $66.4 \pm 34.9$ & $121.0 \pm 87.8$ \\
TTP $\left(\mathrm{mm}^{2}\right)$ & $7.0 \pm 4.2$ & $13.8 \pm 8.8$ & $19.1 \pm 9.1$ \\
Ri $(\mathrm{s})$ & $3.8 \pm 4.4$ & $8.7 \pm 5.3$ & $15.45 \pm 9.3$ \\
\hline
\end{tabular}

mTT: mean transit time; pE: peak enhancement; Ri: rise time, TTP: time to peak; WiAUC: wash-in area under the curve.

In the metastatic lesions after complete ablation (33 lesions), highly significant differences were found for WiAUC $(\mathrm{p}<0.01)$ between the center of the lesion vs. margin.
A

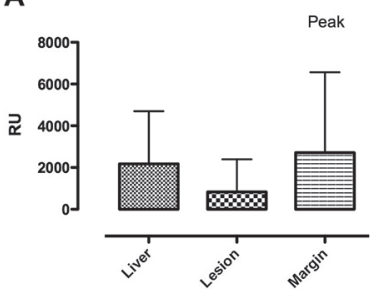

C

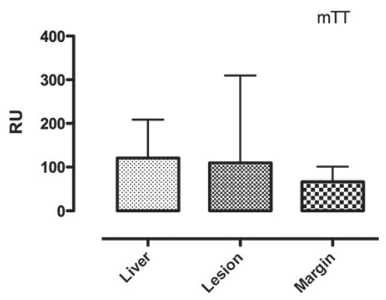

E

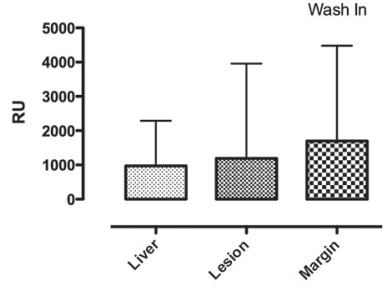

B

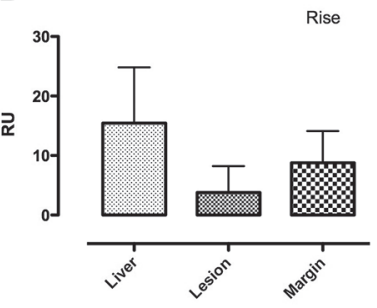

D

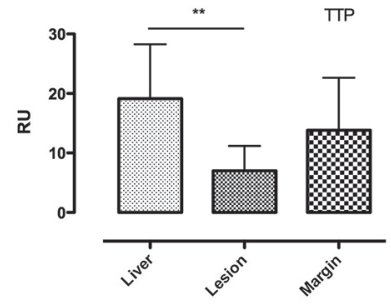

Fig. 4 A-E. Box plots for all perfusion parameters (metastatic lesions with incomplete ablation). No significant differences were found for each of the perfusion parameters $(A-E)$.

Statistically significant differences were also found while comparing the center of the lesion with surrounding liver ( $\mathrm{p}$ $<0.05$ ) regarding WiAUC. $\mathrm{pE}, \mathrm{mTT}$, TTP and Ri showed no significant difference between center of the lesion, margin or surrounding liver (Fig. 5, Table II). The 95\% CIs for differences were 65.58 to 817.5 for center vs. surrounding liver and -955.2 to -203.3 for center vs. margin.

Table II. Perfusion parameters for the ROIs in the center of the lesion, the margin and the healthy liver, acquired within $24 \mathrm{~h}$ following IRE metastases complete ablation.

\begin{tabular}{lccc}
\hline & $\begin{array}{c}\text { Lesion } \\
(\text { mean } \pm \text { SD })\end{array}$ & $\begin{array}{c}\text { Margin } \\
(\text { mean } \pm \text { SD })\end{array}$ & $\begin{array}{c}\text { Surrounding } \\
\text { Liver }( \\
\text { mean } \pm \text { SD })\end{array}$ \\
\hline pE (dB) & $464.3 \pm 1070$ & $5488 \pm 15114$ & $3257 \pm 5275$ \\
WiAUC (-Inf.) & $69.9 \pm 128.9$ & $649.2 \pm 1087$ & $511.4 \pm 689.0$ \\
$\operatorname{mTT}(\%)$ & $221.5 \pm 208.4$ & $210.8 \pm 186.0$ & $155.2 \pm 149.1$ \\
$\operatorname{TTP}\left(\mathrm{mm}^{2}\right)$ & $16.9 \pm 12.8$ & $36.3 \pm 101.9$ & $18.9 \pm 11.5$ \\
$\operatorname{Ri}(\mathrm{s})$ & $34.9 \pm 98.4$ & $20.2 \pm 14.8$ & $18.2 \pm 13.5$ \\
\hline
\end{tabular}

For abbreviations see Table I.

Regarding HCC with incomplete ablation (7 lesions), no significant differences were found for each of the perfusion parameters (Fig. 6, Table III). 


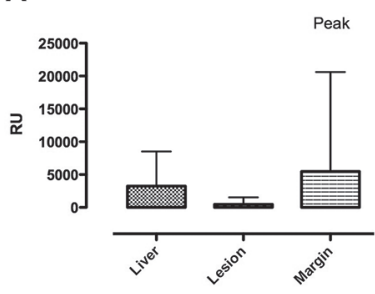

C

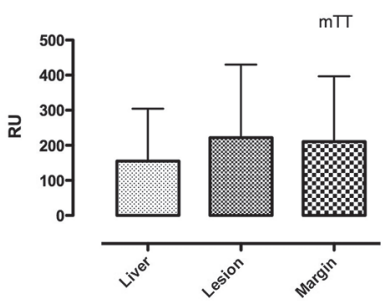

E

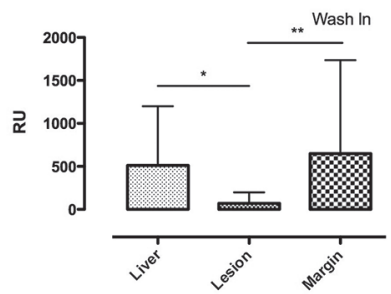

B

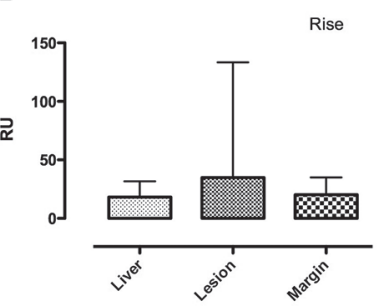

D

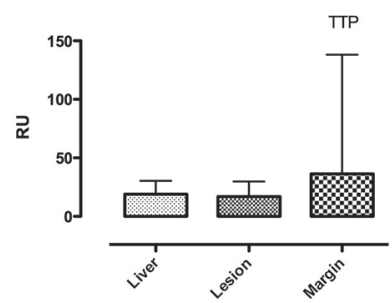

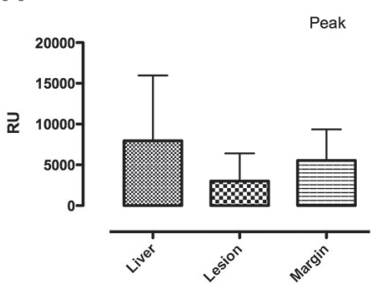

C

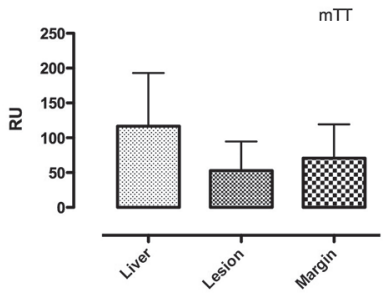

E

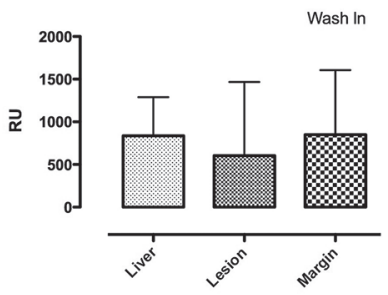

B

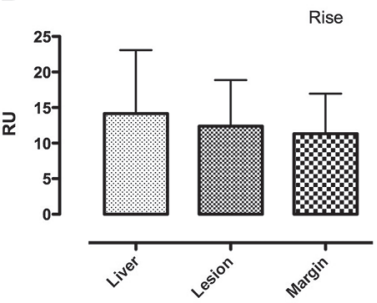

D

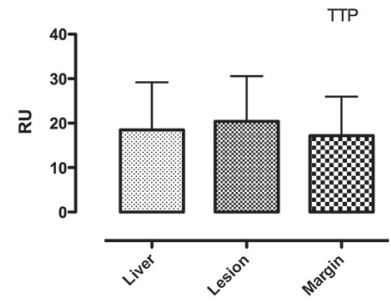

Fig. 5 A-E. Box plots for all perfusion parameters (metastatic lesions with complete ablation) (see Text for details).

Table III. Perfusion parameters for the ROIs in the center of the lesion, the margin and the healthy parenchyma, acquired within $24 \mathrm{~h}$ following IRE - HCC incomplete ablation.

\begin{tabular}{lccc}
\hline & $\begin{array}{c}\text { Lesion } \\
(\text { mean } \pm \text { SD })\end{array}$ & $\begin{array}{c}\text { Margin } \\
(\text { mean } \pm \text { SD })\end{array}$ & $\begin{array}{c}\text { Surrounding } \\
\text { Liver } \\
(\text { mean } \pm \text { SD })\end{array}$ \\
\hline pE (dB) & $3007 \pm 3407$ & $5550 \pm 3795$ & $7963 \pm 8011$ \\
WiAUC (-Inf.) & $605.1 \pm 862.4$ & $849.5 \pm 757.4$ & $836.0 \pm 453.7$ \\
$\operatorname{mTT}(\%)$ & $52.8 \pm 41.7$ & $70.6 \pm 48.7$ & $116.8 \pm 76.4$ \\
TTP $\left(\mathrm{mm}^{2}\right)$ & $20.4 \pm 10.1$ & $17.2 \pm 8.7$ & $18.4 \pm 10.7$ \\
$\operatorname{Ri}(\mathrm{s})$ & $12.3 \pm 6.4$ & $11.3 \pm 5.6$ & $14.2 \pm 8.9$ \\
\hline
\end{tabular}

For abbreviations see Table I.

In the HCC lesions with complete ablation (20 lesions), statistically significant differences were found for WiAUC and $\mathrm{pE}$ between the center of the lesion vs. margin ( $\mathrm{p}<0.05$ for $\mathrm{pE}$ and $\mathrm{p}<0.01$ for WiAUC). Differences between the lesion and surrounding liver were also statistically significant for WiAUC $(\mathrm{p}<0.01)$ and $\mathrm{pE}(\mathrm{p}<0.01)$ (Table IV, Fig. 7). The 95\% CIs of differences regarding $\mathrm{pE}$ were 333.7 to 2118 for center of lesion vs. surrounding liver and -1906 to -121.0 for center of lesion vs. margin. For WiAUC, the $95 \%$ CI of difference were 122.4 to 691.3 for center of lesion vs. surrounding liver and -666.0 to -97.10 for for center vs. margin.

The perfusion software uses pseudo-colors to indicate vascularization. Hypervascularization is shown in red and

Fig. 6 A-E. Box plots for all perfusion parameters (HCC lesions with incomplete ablation). No significant differences were found for each of the perfusion parameters $(\mathrm{A}-\mathrm{E})$.

Table IV. Perfusion parameters for the ROIs in the center of the lesion, the margin and the healthy liver, acquired within $24 \mathrm{~h}$ following IRE - HCC complete ablation

\begin{tabular}{lccc}
\hline & $\begin{array}{c}\text { Lesion } \\
(\text { mean } \pm \text { SD })\end{array}$ & $\begin{array}{c}\text { Margin } \\
(\text { mean } \pm \text { SD })\end{array}$ & $\begin{array}{c}\text { Surrounding } \\
\text { Liver } \\
(\text { mean } \pm \text { SD })\end{array}$ \\
\hline pE (dB) & $34.1 \pm 39.1$ & $415.7 \pm 606.9$ & $441.0 \pm 564.3$ \\
WiAuC (-Inf.) & $605.1 \pm 862.4$ & $849.5 \pm 757.4$ & $836.0 \pm 453.7$ \\
$\operatorname{mTT~(\% )~}$ & $162.4 \pm 147.9$ & $252.6 \pm 190.0$ & $209.9 \pm 175.1$ \\
$\operatorname{TTP}\left(\mathrm{mm}^{2}\right)$ & $18.9 \pm 13.3$ & $18.3 \pm 8.8$ & $20.5 \pm 8.6$ \\
$\operatorname{Ri}(\mathrm{s})$ & $13.9 \pm 11.3$ & $14,8 \pm 8.2$ & $16.0 \pm 8.6$ \\
\hline
\end{tabular}

For abbreviations see Table I.

yellow shades, whereas devascularization is shown in blue and green colors (Fig. 8). In all cases, there was a profound visual reduction of vascularization displayed as blueish and greenish nuances (Fig. 9).

\section{DISCUSSION}

Irreversible electroporation is a relatively new technique for soft tissue ablation relying on electrical fields rather than thermal energy to disrupt the cellular homeostasis thereby causing cell death. The biggest advantage compared to thermal ablation techniques is that theoretically, it only affects the target tissue when properly applied. Critical structures such as 
A

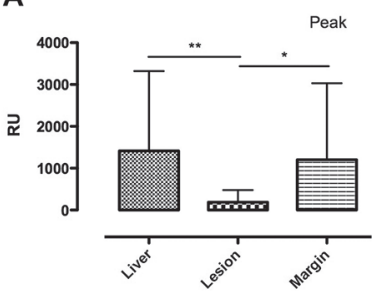

C

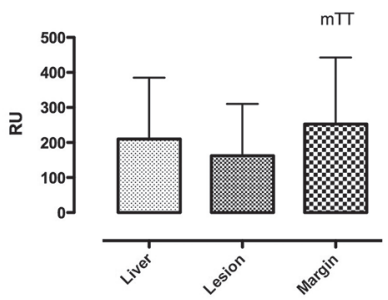

E

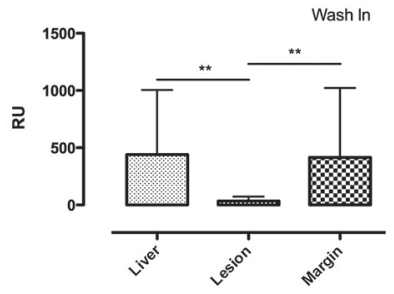

B

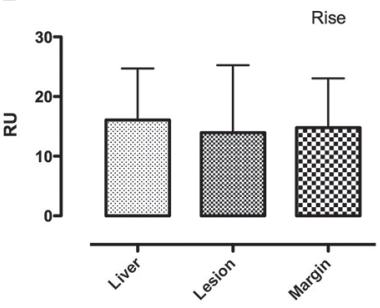

D

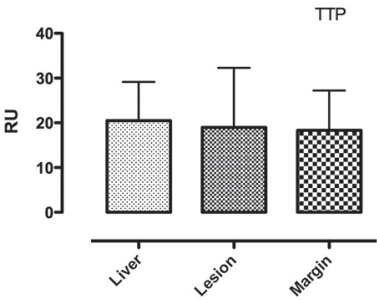

Fig. 7 A-E. Box plots for all perfusion parameters (HCC lesions with complete ablation) (see Text for details).

blood vessels, biliary ducts and nerves are unaffected by this treatment $[13,21]$. This expands the focus of treatable lesions close to major vascular/ biliary/ urinary structures. One major disadvantage of IRE is a considerably prolonged procedure time compared to thermal ablations [15, 22], making general anesthesia inevitable for the intervention and especially there is a remaining vascularization visible after the treatment, which

again makes perfusion analyses more difficult. In some cases, there might still be a remaining suspect vascularization, hence the clinical follow-up is crucial [23].

Studies have reported complication rates of up to $29.3 \%$ [22], significantly higher than for thermal ablations, where complication rates of $6-16 \%$ were observed [24]. Furthermore, the contrast-enhanced imaging characteristics of liver tumors successfully treated by IRE seem different from those following RFA. Unlike RFA, a persistent enhancement of the peritumoral liver parenchyma is found within the IRE ablation zone, and therefore the ablated margin is not clear in IRE. Therefore, CEUS perfusion is not suitable as a sole method for followup of IRE. CEUS allows an exact and continuous evaluation of the capillary microvascularization of tumor lesions with reliable display of an early wash-out and a high sensitivity for detection [25]. Thus, residual tumor can be identified not by arterial hypervascularization but by an early wash-out of suspicious lesions. These are essential factors for identifying residual tumor and for planning re-treatment.

For follow-up evaluation after IRE, evaluation of differences regarding the echogenicity of lesions in conventional ultrasound only offers limited benefits [26]. Hematoma can cause changes of the echogenicity, the air bubbles within the ablation defect lead to attenuation artifacts. Similar problems occur in postinterventional follow-up using MRI and CT. Due to periprocedural bleeding and air bubbles, a reliable differentiation of residual tumor is difficult. Previous studies have found CEUS to be superior to contrast enhanced CT and MRI with a liver specific contrast agent for the diagnosis of residual tumor in the subacute phase following IRE with a sensitivity of $85.7 \%$ vs. $64.3 \%$ and $78.6 \%[18,27]$. The color coded perfusion software $\left(\right.$ VueBox $\left.^{\otimes}\right)$ used in this study shows hyper-enhancement in yellow and red shades, whereas devascularized areas appear blue. By using ROIs in the center of the lesion, the extent of devascularization in the margins and the surrounding liver tissue could be analyzed. The therapeutic success postulates a complete devascularization of the center of the malignant lesion. Nodular enhancement and irregular regional enhancement pattern correlate with the residual vital tumor.

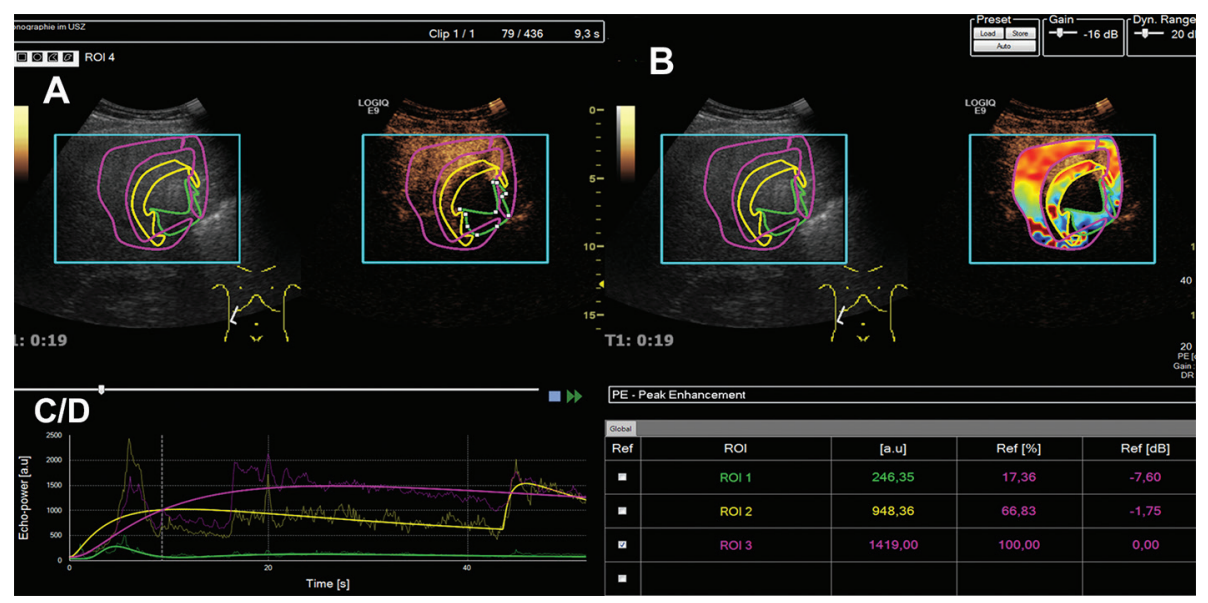

Fig. 8. Case of successful IRE (display of peak enhancement). A. After the intervention, the postembolization defect in CEUS original images appears black, meaning nearly avascular whereas the margin appears partially vascularized. B. In pseudo-colors the defect is shown in blue colors, also showing a devascularization. C/D. TIC analyses show that the perfusion curve for the center of the defect (green) is close to the baseline. 


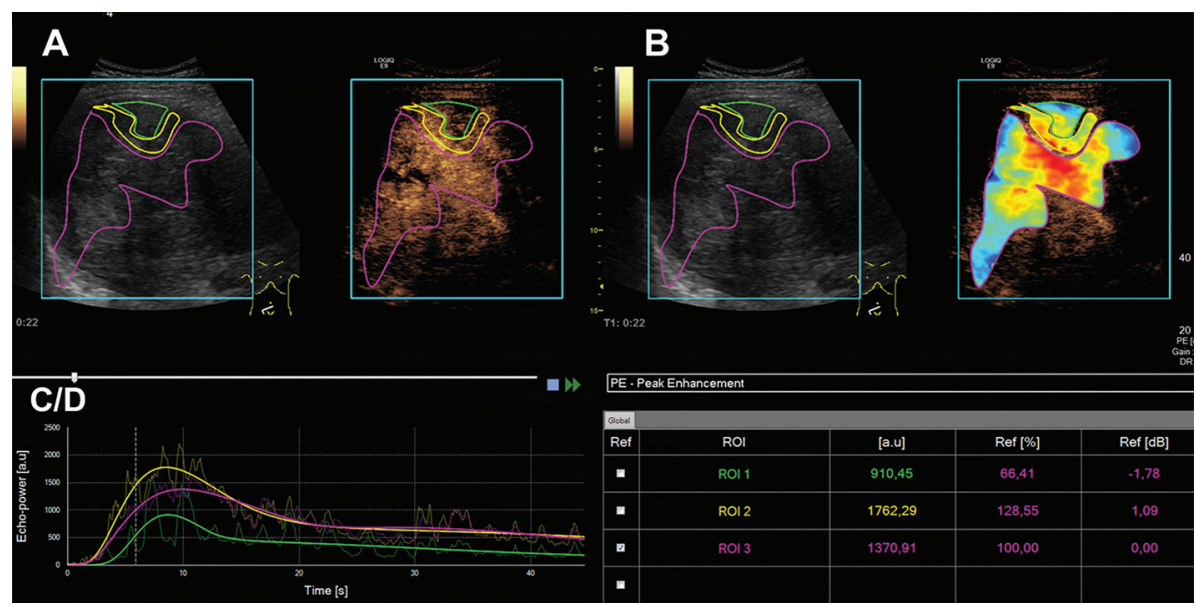

Fig. 9. Case of a partially successful IRE (display of peak enhancement). A. The post-embolization defect in CEUS original images shows residual contrast enhancement, consistent with the remaining tumor. B. In pseudo-colors the defect is shown in blue to green colors, proving an only partial devascularization. C/D. TIC analyses show that the perfusion curve for the center of the defect (green) is not quite close to the baseline confirming a residual tumor.

A critical point is that the perfusion software often shows incomplete devascularization following ablative techniques. Thus, for establishing the clinical success, the short-term follow-up is crucial. In this study, we were able, for the first time, to analyze and compare various perfusion parameters including $\mathrm{pE}$, WiAUC, mTT, Ri, TTP in the center of the lesion, its margins and the surrounding tissue. Our results indicate that focus should be placed upon $\mathrm{pE}$ and WiAUC in future studies, as there are statistically significant differences visible, particularly when comparing the center of the lesion with the margin and the surrounding liver.

Parametric imaging using CEUS can suggest whether ablation therapy using IRE was effective or not. We used various perfusion parameters including $\mathrm{pE}, \mathrm{mTT}$, TTP, Ri and WiAUC. WiAUC is the area under the TIC curve above baseline. It is calculated numerically between the time t 0 and a predefined time tend [20] and is related to the blood volume and thus affected by arterial hypervascularization. Peak enhancement, $\mathrm{Ri}$ and TTP are both wash-in parameters and are crucial for the evaluation of the hypervascularity due to neoangiogenesis in tumoral lesions. It is possible that no differences were found regarding Ri due to the post ablation hyperemia following IRE, The parameter mTT describes the mean time the bubbles need to pass through the ROI and therefore depends upon the tumor type and whether arterial hypervascularity is present or not. In our study, we found slight numeric differences regarding Ri and mTT by comparing center of lesion and margin or surrounding liver. These were, however, not statistically significant, possibly due to the small number of lesions.

For evaluation of an early wash-out, the parameter wash-out time would be required. However, for a sufficient assessment, a continuous scanning of the ablation defect for 3 minutes in metastatic lesions and up to 5 minutes in HCC lesions after contrast application would be necessary. For an adequate evaluation of the entire liver, an additional contrast administration would be required. Therefore, this parameter was not obtained.

In metastatic lesions with complete ablation, perfusion analysis from arterial to portal venous phase showed significant differences regarding WiAUC between the center of the lesion and surrounding liver as well as center of the lesion and margin. If ablation therapy was only partial for metastases and HCCs, no significant differences were detected. This might be, however, due to the very small number of lesions included in each assessment.

In HCC lesions with complete ablation, significant differences were found for $\mathrm{pE}$ and WiAUC while comparing the center of the lesion to margin and surrounding liver. However, evaluation of CEUS contrast dynamics concerning residual nodular tumor lesions with early wash-out is crucial.

Our study has some limitations. The study population was heterogeneous with regard to the tumor sizes, the extent of liver cirrhosis and the underlying malignancy. A further limitation is the relatively small number of patients and lesions in the present study. Therefore, further studies with an increased number of patients are necessary.

\section{CONCLUSION}

CEUS in combination with perfusion analysis is a valuable supporting tool for post-interventional success control following IRE of malignant liver lesions. Special focus should be placed upon WiAUC and $\mathrm{pE}$.

Conflicts of interest: None to declare.

Authors' contributions: E.M.J. collected the data. J.R. and I.W. analyzed the data and drafted the manuscript. A.S. performed the statistics. P.W. and L.P.B performed the ablation therapy. A.S., P.W., C.S., L.P.B., E.M.J. critically revised the manuscript.

\section{REFERENCES}

1. European Association For The Study Of The Liver; European Organisation For Research And Treatment Of Cancer. EASL-EORTC clinical practice guidelines: management of hepatocellular carcinoma. J Hepatol 2012;56:908-943. doi:10.1016/j.jhep.2011.12.001 
2. Mise Y, Sakamoto Y, Ishizawa T, et al. A worldwide survey of the current daily practice in liver surgery. Liver Cancer 2013;2:55-66. doi: $10.1159 / 000346225$

3. Lin SM. Local ablation for hepatocellular carcinoma in taiwan. Liver Cancer 2013;2:73-83. doi:10.1159/000343843

4. Chan SC. Liver transplantation for hepatocellular carcinoma. Liver Cancer 2013;2:338-344. doi:10.1159/000343849

5. Raoul JL, Gilabert M, Piana G. How to define transarteria chemoembolization failure or refractoriness: a European perspective Liver Cancer 2014;3:119-124. doi:10.1159/000343867

6. Kudo M, Matsui O, Izumi N, et al. JSH Consensus-Based Clinical Practice Guidelines for the Management of Hepatocellular Carcinoma: 2014 Update by the Liver Cancer Study Group of Japan. Liver Cancer 2014;3:458-468. doi:10.1159/000343875

7. Ma R, Feng Y, Lin S, et al. Mechanisms involved in breast cancer liver metastasis. J Transl Med 2015;13:64. doi:10.1186/s12967-015-0425-0.

8. Adam R, De Gramont A, Figueras J, et al. The oncosurgery approach to managing liver metastases from colorectal cancer: a multidisciplinary international consensus. Oncologist 2012;17:1225-1239. doi:10.1634/ theoncologist.2012-0121

9. Schoellhammer HF, Hsu F, Vito C, et al. Complete pathologic response of HER2-positive breast cancer liver metastasis with dual anti-HER2 antagonism. BMC Cancer 2014;14:242. doi:10.1186/1471-2407-14-242

10. Foss A, Lerut JP. Liver transplantation for metastatic liver malignancies. Curr Opin Organ Transplant 2014;19:235-244. doi:10.1097/ MOT.0000000000000086

11. Townsend AR, Chong LC, Karapetis C, Price TJ. Selective internal radiation therapy for liver metastases from colorectal cancer. Cancer Treat Rev 2016;50:148-154. doi:10.1016/j.ctrv.2016.09.007

12. Zorbas G, Samaras T. A study of the sink effect by blood vessels in radiofrequency ablation. Comput Biol Med 2015;57:182-186. doi:10.1016/j.compbiomed.2014.12.014

13. Davalos RV, Mir IL, Rubinsky B. Tissue ablation with irreversible electroporation. Ann Biomed Eng 2005;33:223-231. doi:10.1007/ s10439-005-8981-8

14. Ahmed M, Goldberg SN. Thermal ablation therapy for hepatocellular carcinoma. J Vasc Interv Radiol 2002;13:S231-S244.

15. Niessen C, Beyer LP, Pregler B, et al. Percutaneous Ablation of Hepatic Tumors Using Irreversible Electroporation: A Prospective Safety and Midterm Efficacy Study in 34 Patients. Vasc Interv Radiol 2016;27:480486. doi:10.1016/j.jvir.2015.12.025

16. Cannon R, Ellis S, Hayes D, Narayanan G, Martin RC 2nd. Safety and early efficacy of irreversible electroporation for hepatic tumors in proximity to vital structures. J Surg Oncol 2013;107:544-549. doi:10.1002/jso. 23280
17. Wiggermann P, Puls R, Vasilj A, et al. Thermal ablation of unresectable liver tumors: factors associated with partial ablation and the impact on long-term survival. Med Sci Monit 2012;18:CR88-CR92. doi:10.12659/ msm. 882463

18. Sugimoto K, Moriyasu F, Saito K, Kobayashi Y, Itoi T. Multimodality imaging to assess immediate response following irreversible electroporation in patients with malignant hepatic tumors. J Med Ultrason (2001) 2017;44:247-254. doi:10.1007/s10396-016-0767-0

19. Rennert J, Wiesinger I, Schicho A, et al. Color coded perfusion imaging with contrast enhanced ultrasound (CEUS) for post-interventional success control following trans-arterial chemoembolization (TACE) of hepatocellular carcinoma. PLoS One 2019;14:e0217599. doi:10.1371/ journal.pone.0217599

20. Dietrich CF, Averkiou MA, Correas JM, Lassau N, Leen E, Piscaglia F. An EFSUMB introduction into Dynamic Contrast-Enhanced Ultrasound (DCE-US) for quantification of tumour perfusion. Ultraschall Med 2012;33:344-351. doi:10.1055/s-0032-1313026

21. Dollinger M, Müller-Wille R, Zeman F, et al. Irreversible Electroporation of Malignant Hepatic Tumors--Alterations in Venous Structures at Subacute Follow-Up and Evolution at Mid-Term Follow-Up. PLoS One 2015;10:e0135773. doi:10.1371/journal.pone.0135773

22. Philips P, Hays D, Martin RC. Irreversible electroporation ablation (IRE) of unresectable soft tissue tumors: learning curve evaluation in the first 150 patients treated. PLoS One 2013;8:e76260. doi:10.1371/ journal.pone.0076260

23. Wiesinger I, Wiggermann P, Zausig N, et al. Perkutane Therapie maligner Leberläsionen: Evaluation des Therapieerfolgs mittels CEUS und Perfusionssoftware. Ultraschall Med 2018;39:440-447. doi:10.1055/s-0043-119353

24. Poon RT, Ng KK, Lam CM, et al. Learning curve for radiofrequency ablation of liver tumors: prospective analysis of initial 100 patients in a tertiary institution. Ann Surg 2004;239:441-449. doi:10.1097/01. sla.0000118565.21298.0a

25. Sporea I, Săndulescu DL, Şirli R, et al. Contrast-Enhanced Ultrasound for the Characterization of Malignant versus Benign Focal Liver Lesions in a Prospective Multicenter Experience - The SRUMB Study. J Gastrointestin Liver Dis 2019;28:191-196. doi:10.15403/jgld-180

26. Lin MX, Kuang M, Xu M, et al. Ultrasound and Contrast-Enhanced Ultrasound for Evaluation of Irreversible Electroporation Ablation: In Vivo Proof of Concept in Normal Porcine Liver. Ultrasound Med Biol 2016;42:2639-2649. doi:10.1016/j.ultrasmedbio.2016.07.007

27. Niessen C, Beyer LP, Haimerl M, et al. Percutaneous irreversible electroporation of hepatocellular carcinoma: Contrast-enhanced ultrasound-findings during 1-year follow-up. Clin Hemorheol Microcirc 2019;72:85-93. 